\title{
Pulmonary sinus of Valsalva aneurysm: A rare entity
}

Trushar Gajjar, Nageswar Rao, Neelam Desai

Sri Sathya Sai Institute of Higher Medical Sciences - Prasanthigram, CTVS Department, Prasanthigram, Andhra Pradesh, India

Received: September 26, 2016 Accepted: January 07, 2017 Published online: April 17, 2017

\section{ABSTRACT}

Pulmonary sinus of Valsalva aneurysm is rare. An intrinsic weakness of the wall and increased hemodynamic stress is the main causative factor. Surgery has a definite role. Herein, we describe a case of pulmonary sinus of Valsalva aneurysm who was successfully treated in our clinic.

Keywords: Pulmonary sinus of Valsalva aneurysm; pulmonary valve; sinus of Valsalva aneurysm.

Pulmonary artery sinus of Valsalva aneurysm is an unusual lesion, ${ }^{[1-4]}$ which can be associated with congenital heart diseases, pulmonary artery hypertension, pulmonary valve stenosis, connective tissue diseases (such as Marfan syndrome), and vasculitis. To the best of our knowledge, true pulmonary sinus of Valsalva aneurysm with a dilated pulmonary artery has not been reported in the literature. Herein, we describe a case of pulmonary sinus of Valsalva aneurysm who was successfully treated in our clinic.

\section{CASE REPORT}

A five-month-old boy presented with a complaint of recurrent respiratory tract infection. Chest X-ray showed a dilated pulmonary artery (Figure 1). Echocardiography showed a large perimembranous ventricular septal defect (VSD), large-sized patent ductus arteriosus (PDA), a dilated main pulmonary artery and branches. Cardiac catheterization showed a right ventricular systolic and mean pulmonary artery pressure of $67 \mathrm{mmHg}$ and $43 \mathrm{mmHg}$, respectively, and a left-to-right shunt at a ratio of 2.1:1 and a pulmonary vascular resistance index of 2.4 Woods units. Angiography showed a dilated pulmonary artery with an aneurysm of the anterior sinus of Valsalva (Figure 2). The patient underwent a successful closure of VSD and PDA with plication of the sinus of Valsalva aneurysm (Figure 3, 4). The post operative course in the hospital was uneventful and was discharged in stable condition on sixth postoperative day.

\section{DISCUSSION}

In the literature review, true pulmonary sinus of Valsalva aneurysm is rare and unusual lesion. ${ }^{[1-4]}$ This anomaly can be seen with associated with

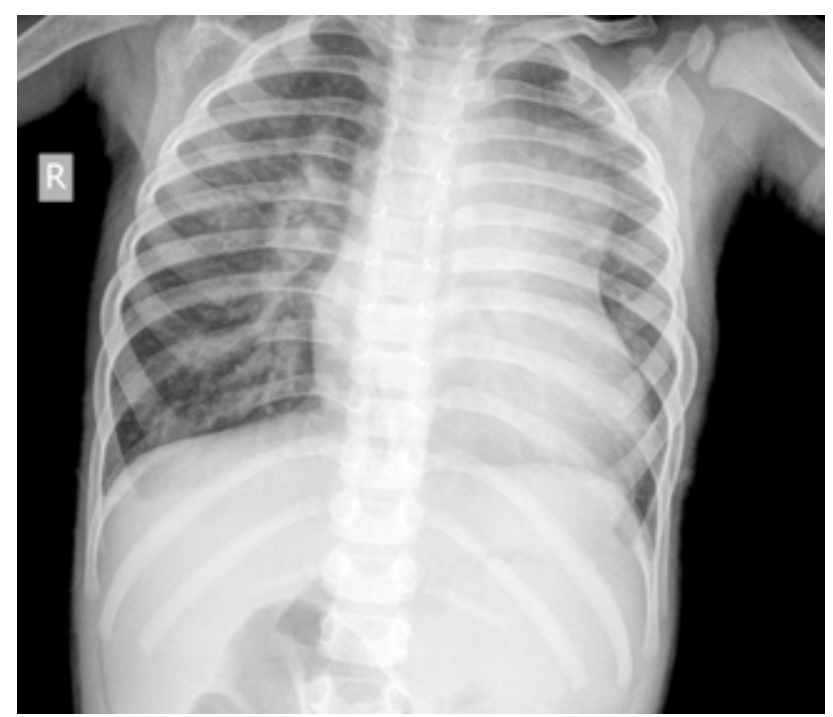

Figure 1 . A chest $\mathrm{X}$-ray image showing a dilated pulmonary artery with plethora.

Corresponding author: Trushar Gajjar, MD. Sri Sathya Sai Institute of Higher Medical Sciences - Prasanthigram, CTVS Department, Prasanthigram, 515134 Andhra Pradesh, India.

Tel: +91 9700355411 e-mail: trushargajjar@gmail.com 


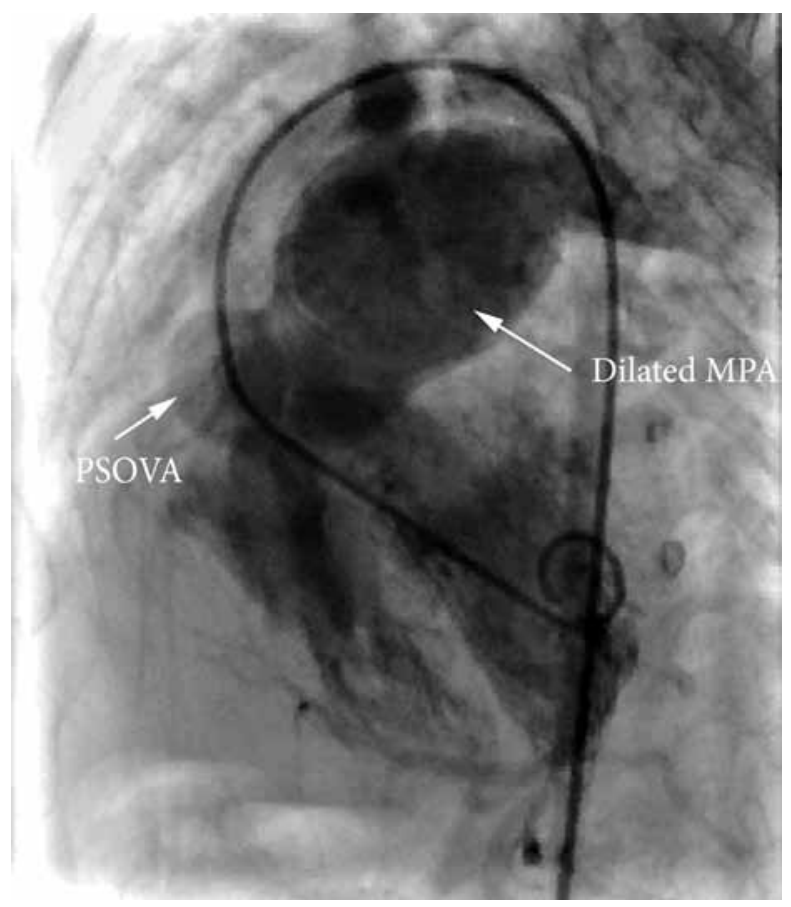

Figure 2. A left ventriculogram image in left anterior oblique cranial view showing VSD with a dilated MPA and an aneurysm of anterior sinus of Valsalva of pulmonary artery.

VSD: Ventricular septal defect; MPA: Main pulmonary artery.

congenital heart disease, pulmonary arterial hypertension, valvar pulmonary stenosis, connective tissue disorders and vasculitis. Other causes include infections (i.e., tuberculosis, syphilis), atherosclerosis, hypertension, hereditary hemorrhagic telangiectasia, cystic media necrosis, and traumas. ${ }^{[1]}$ The pathological

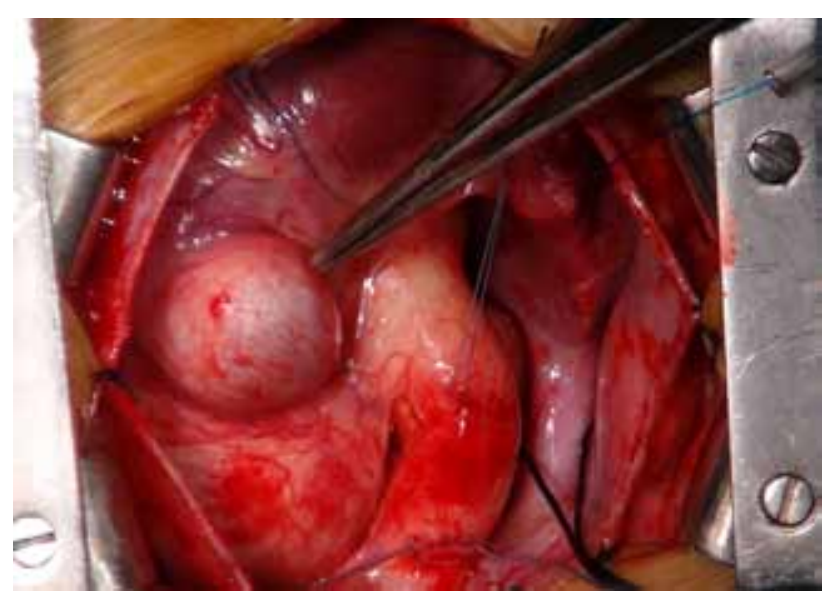

Figure 3. An intraoperative view showing a dilated main pulmonary artery with an aneurysm of sinus of Valsalva. cause is intrinsic weaknesses of the arterial wall in combination with an increased hemodynamic stress are responsible for its formation. ${ }^{[2-4]}$ The clinical manifestations are mostly non-specific and symptoms are usually due to associated lesions. ${ }^{[2]}$ Cardiac catheterization and angiography are the gold standards for the diagnosis; however, noninvasive imaging methods including spiral computed tomography angiography and magnetic resonance imaging are also useful tools. ${ }^{[3,4]}$ Surgical intervention is often recommended to symptomatic patients and to those with underlying diseases or complications, left-to-right shunts, pulmonary arterial hypertension, and large-sized aneurysms. ${ }^{[4]}$ In the treatment of lowpressure pulmonary artery aneurysms, intervention is required when the right ventricular size and function alter due to pulmonary regurgitation or pulmonary stenosis. However, asymptomatic, smallsized aneurysms need close follow-up, as the risk of rupture is low, while these lesions requires an intervention if patient is undergoing open heart surgery for associated lesion. ${ }^{[4]}$

In conclusion, pulmonaty sinus of Valsalva aneurysm is an unusual entity mostly associated with other congenital anomaly. The weakness of arterial wall with increased hemodynamic stress leads to this anomaly. Diagnosis can be achieved by conventional echocardiography and angiography. The computed

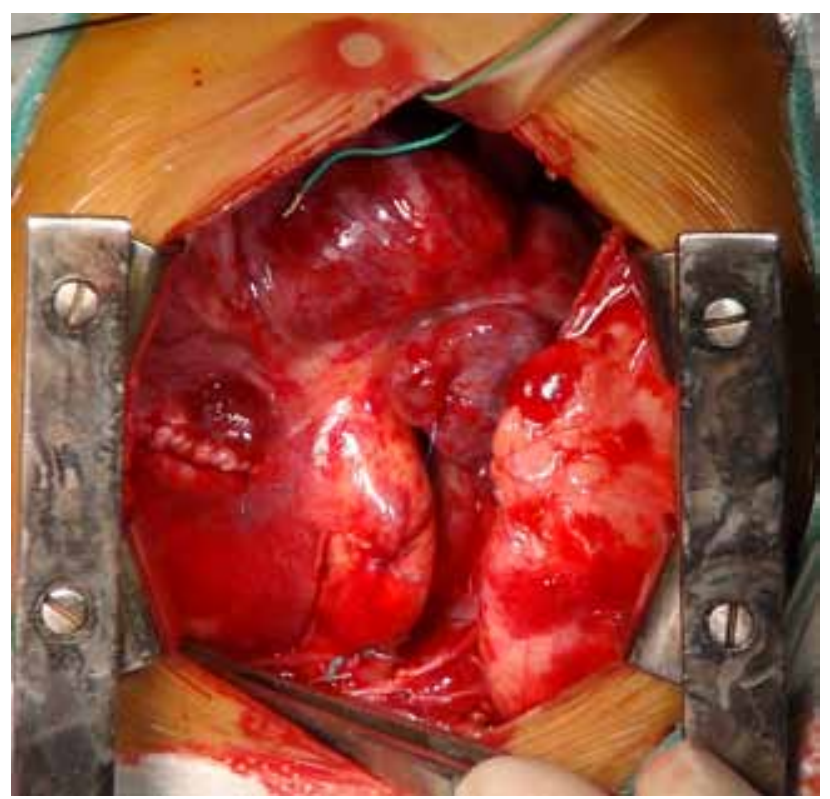

Figure 4. An intraoperative view showing repaired aneurysm of sinus of Valsalva. 
tomography angiography and magnetic resonance imaging helps in better delineation of anatomy. Small and asymptomatic aneurysm needs observation while associated congenital heart disease and large aneurysm needs surgical intervention.

\section{Declaration of conflicting interests}

The authors declared no conflicts of interest with respect to the authorship and/or publication of this article.

\section{Funding}

The authors received no financial support for the research and/or authorship of this article.

\section{REFERENCES}

1. Bartter T, Irwin RS, Nash G. Aneurysms of the pulmonary arteries. Chest 1988;94:1065-75.

2. Butto F, Lucas RV Jr, Edwards JE. Pulmonary arterial aneurysm. A pathologic study of five cases. Chest 1987;91:237-41.

3. Senbaklavaci O, Kaneko Y, Bartunek A, Brunner C, Kurkciyan E, Wunderbaldinger $\mathrm{P}$, et al. Rupture and dissection in pulmonary artery aneurysms: incidence, cause, and treatment--review and case report. J Thorac Cardiovasc Surg 2001;121:1006-8.

4. Veldtman GR, Dearani JA, Warnes CA. Low pressure giant pulmonary artery aneurysms in the adult: natural history and management strategies. Heart 2003;89:1067-70. 